\title{
Development and Applications of the UAL-based SNS Ring Simulation Environment*
}

\author{
N.Malitsky, P.Cameron, A.V.Fedotov, J.Smith, J.Wei \\ BNL, Upton, NY 11973, USA
}

\begin{abstract}
The SNS Ring off-line parallel simulation environment based on the Unified Accelerator Libraries (UAL) has been implemented and used for extensive full-scale beam dynamics studies arising in high-intensity rings. The paper describes the structure of this environment and its application to various high-intensity topics and diagnostics modeling.
\end{abstract}

\section{INTRODUCTION}

Designs and parameters of modern high-intensity machines, such as the SNS accumulator ring, impose new expectations on the beam dynamics studies. One of the major scientific and technical challenges is the extremely strict requirement on uncontrolled beam loss at $10^{-4}$ level. In order to describe and analyze such low-level losses, one should closely reproduce all actual effects of a realistic machine. For addressing this task, we have employed the open simulation environment of the Unified Accelerator Libraries (UAL[1-2]). When we started this project, UAL had been already applied to several accelerators. However, unlike other UAL applications, the SNS Ring required a new combination of collective physical effects and dynamic processes. Then the new SNS-specific package has been implemented. Its development included the integration of the UAL infrastructure with three accelerator libraries (ORBIT, ACCSIM, and MB), deployment of this infrastructure on the MPI-based parallel facility, and the implementation of the injection framework. In the paper we describe the structure of the SNS simulation package and its application to complex beam dynamics studies and diagnostics modeling.

\section{UNIFIED ACCELERATOR LIBRARIES}

The Unified Accelerator Libraries (UAL) is an accelerator simulation environment aiming to develop the realistic beam dynamics models including an unlimited combination of physical effects and dynamic processes. The goal has been achieved by introducing the open infrastructure where diverse accelerator approaches and programs are implemented as collaborative $\mathrm{C}++$ libraries connected together via Common Accelerator Objects (such as Element, Twiss, Particle, etc.). At this time, the UAL 1.x off-line simulation environment joins six objectoriented accelerator programs (see Fig.1). A universal homogeneous interface based on the Perl extension mechanism allows scientists to combine and manage all these $\mathrm{C}++$ and Perl components in the single development and research environment. The high-level applications are built on the top of the project-specific Shell (or Façade) that consolidates and facilitates access to numerous lowlevel UAL interfaces and data containers.

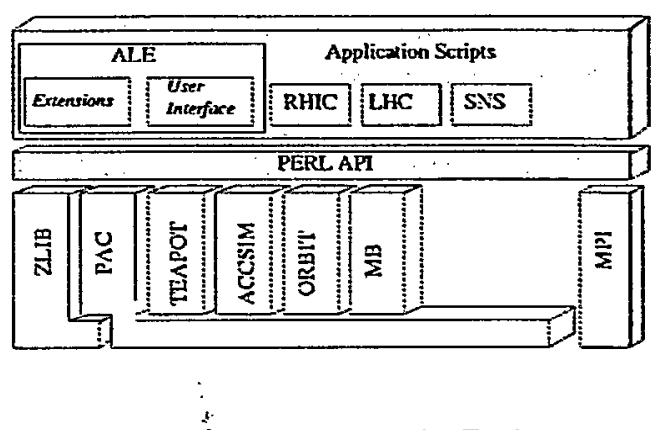

FIGURE 1. Unified Accelerator Libraries Environment.

The same UAL infrastructure fits to the MPI-based parallel environment and has been recently deployed on the parallel cluster for efficient high-intensity beam dynamics modeling. Parallel versions of the time consuming algorithms (such as collective space charge effects) are implemented as ordinary $\mathrm{C}++$ shared libraries and can be mixed together with other UAL sequential and/or parallel modules.

\section{SNS RING SIMULATION ENVIRONMENT}

According to the UAL approach, the SNS simulation environment has been organized as an additional package integrating together SNS-specific modules and extensions. The overall architecture of the SNS environment is illustrated in Figure 2. A physicist interacts with the SNS::Shell interface that encompasses a set of high-level MAD-like or TEAPOT-like commands. Most of these commands are project-independent, and inherited from the common class ALE::Shell. The SNS Ring multi-turn injection scenario is considered as an

*Work performed under the auspices of the U.S. Department of Energy. 
extension of the present UAL environment and implemented directly in the SNS::Shell class.

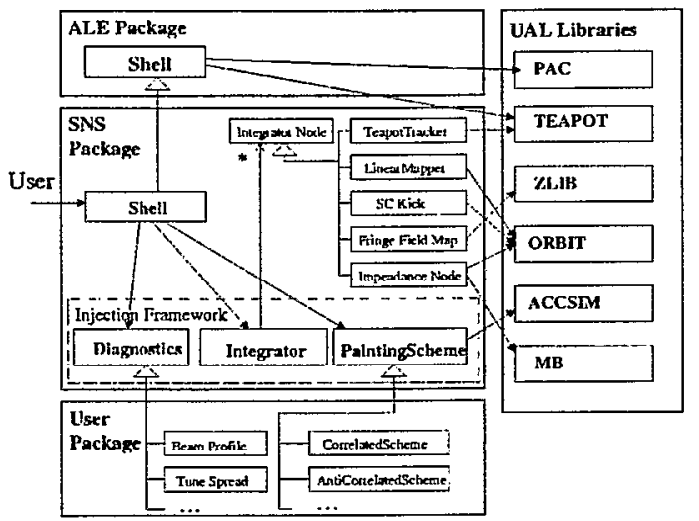

FIGURE 2. Structure of the SNS ring simulation package.

To facilitate the development and study of the SNS Ring applications, we have implemented the injection framework with three basis classes: SNS::Integrator, SNS::PaintingScheme, and SNS::Diagnostics. Each basis class originates an open collection of alternative approaches that can be selected in any combination with the instances of two other collections.

\section{BEAM DYNAMICS APPLICATIONS}

One of the major advantages of the SNS package is its capability to support comprehensive studies of various effects, as well as a complex combination of several effects in the single simulation environment. Below, we list some of the beam dynamics topics to which the SNS package was applied:

- Single-particle tasks, including such effects as kinematic non-linearity, non-linear tune-spread, dynamic aperture, and resonance driven diffusion maps [3]-[4].

- Effect of space charge during transverse painting [5].

- Optimization of painting bump functions [6].

- Combined tune spread due to the space charge, chromaticity and other nonlinearities [6]-[7].

- Imperfection resonance crossing in the presence of space charge with corresponding choice of working points and intensity limitation [7]-[8].

- Effect of $1 / 2$ coherent resonance crossing in the presence of high-order resonances.

- Coherent resonance crossing of coupling resonances.

- Collective instability due to the transverse impedance [9].

An ability to study a complex combination of several effects enables us to have a realistic expectation for beam losses and intensity limitation. Here, we demonstrate the resonance driven beam loss for the two working points of the SNS: $(6.23,6.20)$ and $(6.4,6.3)$. The imperfection errors are excited at a level slightly higher than expected to get a conservative estimate. The full 1060-turn injection is then performed for each of beam intensities with beam losses at the end of accumulation recorded for a specific acceptance. Figures 3-4 and 5-6 show the tune spreads and corresponding resonance driven loss curves for both working points.
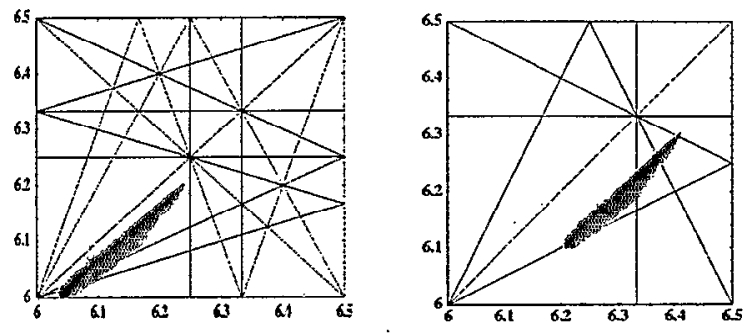

FIGURE 3-4. Tune spreads for working points $(6.23,6.20)$ and $(6.4,6.3)$, respectively.

The working point $(6.23,6.20)$ is essentially free from resonance losses apart from some low loss due to the resonances above the working point and chromatic tune spread. Here, the first-turn losses due to injection and $\mathrm{H}^{-}$ stripping are omitted from the loss curve. For high beam intensities the tune are effectively depressed by the space charge. The only resonances up to the $4^{\text {th }}$ order, which beam crosses during accumulation, are the difference resonances. As a result of the space charge coupling, the beam with approximately similar transverse beam emittances is not susceptible to the difference resonance. The intensity limitation for this working point is associated with the coherent beam response near the tune of 6.0 , which limits beam intensity to slightly above $\mathrm{N}=2 * 10^{14}$ at the energy of $1 \mathrm{GeV}$ [10]. However, this limitation is due to the structure resonances and thus is very strict.

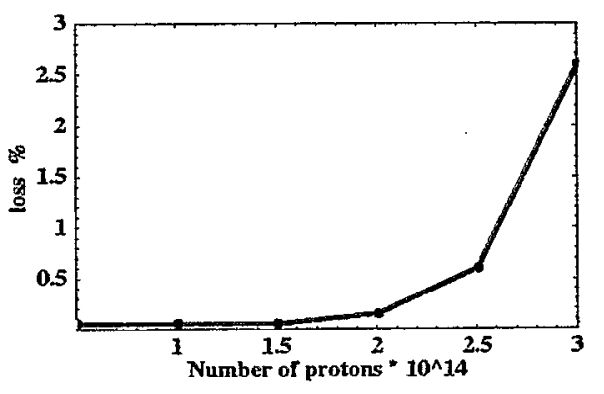

FIGURE 5. Loss curve for the working point $(6.23,6.20)$

For the working point $(6.4,6.3)$, the loss curve demonstrates impact of each individual resonance crossed during accumulation. The strong loss at low intensity is due to the sum sextupole resonance at which the working be slightly adjusted to avoid this strong resonance loss. Other loss peaks are due to the $3^{\text {rd }}$ and $4^{\text {th }}$ order resonances, which are crossed for higher beam intensity. T'he losses due to these resonance are very strong, however, they are all imperfection resonances. With 
appropriate correction schemes, which are available in the SNS, one can attempt to compensate such resonances. As, a result, with successful compensation, the real loss for this working point happens only at a very high intensity due to the coherent beam response to the. structure resonances, as shown in Fig. 6.

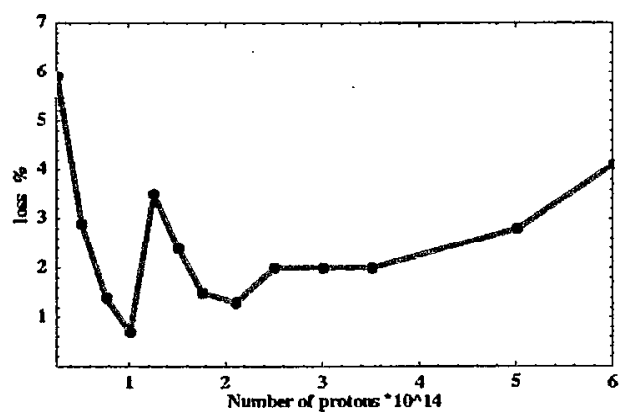

FIGURE 6. Loss curve for the working point $(6.3,6.4)$

\section{TUNE MEASUREMENT MODELING}

Minimization of beam loss and activation in the Spallation Neutron Source Ring is highly dependent on proper control of the tune footprint. The major contributors to tune spread are space charge, chromaticity, and uncompensated fringe fields. In addition to the challenge of accurate measurement in the presence of large tune spread, large dynamic range will be required to permit measurement through the accumulation cycle. There are many possibilities for measuring coherent and incoherent tune and tune shift in the SNS Ring. These include [11]:

- Coherent (dipole) tune/tune shift from impulse excitation

- Incoherent tune from injection oscillations.

- Incoherent tune from Schottky.

- Incoherent tune from quadrupole mode oscillation.

- Incoherent tune from resonance crossing.

- Incoherent tune from Beam Transfer Function.

To facilitate the design of tune measurement systems, we have been employing the same simulation model used in the SNS Ring beam dynamics studies. The Beam Transfer Function (BTF) approach is its first application. Following the UAL generic scheme, the BTF measurement has been implemented as another tracking scenario with two new diagnostics elements: kicker and BPM. The kicker excites the propagated bunch during each turn of the injection painting and delegates it to the next tracking module. The BPM element calculates the center of bunch and writes the turn-by-turn data into the external file. This application allows one to measure the BPM response to the kicker deflection at a single predefined frequency. Then the tune footprint measurement is represented by a set of several simulation runs, each with a different predefined excitation frequency. Values of FFT signals determine the boundary of the tune footprint. Figure 7 shows preliminary FFT results for the tune spread due to chromaticity.

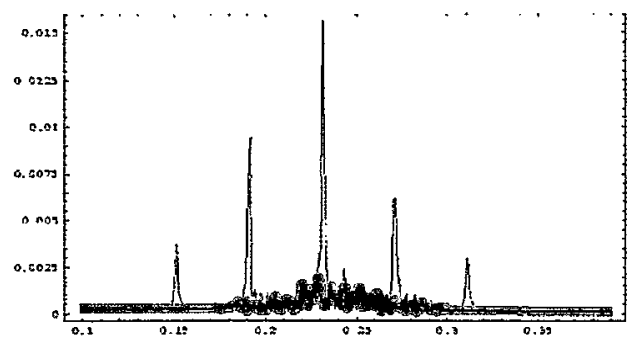

FIGURE 7. Model-based 'measurement' of the tune spread due to chromaticity.

These preliminary results confirm the measurement approach. The method is now being applied to measure full non-linear tune footprint with space charge. To facilitate future activities, the model will be enhanced with the new very efficient tracking engine and additional diagnostics extensions. These calculations were done at baseband with a non-resonant pickup, when in fact the kicker and pickup will operate at about $50 \mathrm{MHz}$, and the pickup $Q$ will approach 100 . Kicks in the above modeling were about a factor of 100 stronger than what will be required with the resonant pickup. The observed BTF at $50 \mathrm{MHz}$ will show broadening of the revolution line due to a comparatively large relativistic slip factor $\eta$, as well as asymmetric chromatic broadening of the betatron sidebands.

\section{REFERENCES}

1. N.Malitsky and R.Talman, AIP 391 (1996).

2. N. Malitsky and R.Talman, ICAP 98 (1998).

3. A.V. Fedotov et al., Proc. of EPAC'00, p.1492.

4. I. Papaphilippou, Proc. of PAC'01 (Chicago), p.462.

5. A.V. Fedotov et al., Proc. of PAC'01, p. 2851.

6. A.V. Fedotov, SNS project ASAC reviews (2001-02).

7. A.V. Fedotov et al., Proc. of PAC'01, p. 2848.

8. A.V. Fedotov, N. Malistky and J. Wei, BNL/SNS Tech Note $086(2001)$.

9. A.V. Fedotov et al., "Exploring transverse beam stability in the SNS in the presence of space charge", EPAC'02 (to be published).

10. A.V. Fedotov and I.Hofmann, "Half Intger resonance crossing and space charge limit", EPAC"02 (to be published).

11. P.Cameron, A.Fedotov, and N.Malitsky, "Tune Measurement in the SNS Ring", EPAC'02 (to be published). 